\title{
Spatial Analysis and Perspective of Flaming Transition through Images
}

\author{
Sambit Supriya Dash ${ }^{1}$, Rahul Ravichandran ${ }^{1}$, Vikram Ramanan ${ }^{2}$ and Vinayak Malhotra ${ }^{1}$ \\ ${ }^{1}$ Department of Aerospace Engineering, SRM Institute of Science and Technology \\ Chennai, India \\ sambitd0@gmail.com; rahulravi3097@gmail.com; vinayakmalhotra.m@ktr.srmuniv.ac.in \\ ${ }^{2}$ Department of Aerospace Engineering, Indian Institute of Technology Madras \\ Chennai, India \\ vikrambest@yahoo.co.in
}

\begin{abstract}
The Combustion process in propulsion systems comprises of premixed flames and diffusion flames. As an active chemical propulsion system, the efficiency of operations depends significantly upon the type of combustion. In real cases, the transition of flames from one mode to another is noted in wide range of cases. This has necessitated research efforts to fundamentally understand the phenomenon for better combustion and thus better propulsion. The combustion transition from pre-mixed to the diffusion state is one of the least explored and quantitatively analysed domains. Present work, experimentally attempts to extensively study and quantify the physical causes thoroughly through manually induced transition in a butane-based cylinder fitted with a nozzle by using image processing techniques. The manually induced transition is processed by varying the inlet area for air entrainment. The phenomenon is systematically video-graphed and image segmentation is carried out to acquire the desired characters. These methods are implemented in unison to theoretically establish a non-dimensional parameter associated with the transition process and thus characterizing it. The results obtained from this experiment could be easily applied to liquid propellant-based propulsion systems for terrestrial and outer space utilities, where micro-gravity conditions play the cardinal role in combustion physics. Results acquired from the experiments are expected to be very helpful in application to space propulsion systems by yielding a prospective insight into the subject, leading to significant improvement in the efficiency and Eco-friendliness of existing propulsion systems.
\end{abstract}

Keywords: Premixed Flames, Transition, Diffusion Flames, Enclosure Area, Flame Length.

\section{Introduction}

Combustion primarily represents an exothermic chemical reaction by using fuel and oxidizer associated with a reaction zone. Fuel and oxidiser mix in required proportions and combustion occurs through an intricate sequence of reaction steps. Combustion is critically important and the resultant energy interactions and governing phenomenon sources close to $80 \%$ of the world energy requirements in form of like propulsion, heating, electricity production. This branch of physics is broadly classified into smoldering and flaming. The classification is based on the manifestation of flames resulting from highly exothermic reactions yielding high flame temperatures depending on the fuel-oxidizer ratio. Different types of flames can result from the way in which the fuel and oxidant are mixed. Combustion can occur in premixed or diffusion modes. Diffusion flame represents diffusion-controlled combustion with diffusion rate greater than the reaction rate. In diffusion flame, fuel and oxidiser concentration vanishes at flame front and the flame region is very thin. In premixed flames, fuel such as natural gas, commercial and industrial liquid fuels, usually termed fuel oils and air mixture kept in an open tube is lighted by a spark and the propagation of the flame is observed at a certain velocity. In comparison to the premixed flames (short and blue), diffusion flames are longer and yellow with higher stability range, luminosity, sooty and flame temperature is not very high. When the fuel flow rate is relatively low, the incoming gaseous flow of fuel and air is laminar, as is the flame however, high fuel flows may lead them to being turbulent. Thus, flames are generally identified as to be laminar premixed, laminar diffusion, turbulent premixed or turbulent diffusion. In addition, they can also be categorized into stationary or propagating flames. Partially premixed flames contain a rich premixed fuel-air mixture in a stream, and, for complete combustion to occur, they require the transport of oxidizer from an appropriately oxidizer rich mixture that is present in another stream. The stationary flames are widely used in domestic or industrial burners, the propagating flames being involved in explosions. An important aspect of the combustion research is investigation of the behaviour of flames in response to numerous parametric variations and external influences. The premixed and diffusion 
flames had been extensively studied and explored owing to the strong physical presence and applications. Combustion systems are one of the most important and complex highlighting an interesting aspect of the presence of partially flames as a coupled resentment between premixed flame and diffusion flame.

Flames in most practical applications cannot be described as purely premixed or non-premixed. The phenomenon observes a phase of transition in-between. The transition from laminar to turbulent flame takes place as the flow velocity increases. Understanding of the flaming transition is important from both practical and scientific considerations because of their widespread occurrence in many applications including gas-fired domestic burners, industrial furnaces, Bunsen burners, turbulent combustion and spray flames. Owing to the wide range of applications from engineering systems of heat, power generation, transportation systems, significant performance losses and fire disasters are noted with flames behaviour being uncertain and turbulent in nature.

Following the classical work of Damkohler [1] on jet flames, appreciable experimental, analytical, numerical work had been done to advance the fundamental understanding of governing physics. Thomas et. al., [2] investigated the buoyant diffusion flames with some measurements of air entrainment, heat transfer, and flame merging. The total quantity of air below the mean flame height was approximately one order times the stoichiometric requirements, a substantial part of the air flowing upwards around the flame. The total flow exceeded that estimated from entrainment theory and measurements of flame tip velocity. The convection transfer above the fuel surface was found to be about $1 / 5^{\text {th }}$ of the total heat transfer at the centre increasing to about $1 / 2$ at the edge. Elementary considerations of entrainment and the motion of flames were applied to the merging of the flames and a reasonable agreement between theory and experiment was found. Lin and Sohrab [3] explored transition from premixed to diffusion flames in a conserved system by shifting the reactants from one side to another in counter flow configuration and identified three types of transition. The investigation suggested that changes in flame structure resulting from transition could provide a better understanding about the equilibrium state. The study also suggested the fact that the transition of Diffusion Flame to Premixed Flame is always accompanied by aerodynamic modification of the flow field. This is a consequence of the reaction stoichiometry as well as the different molecular weights of the fuel and oxidizer. Same burning intensities of the Diffusion and the Premixed Flame were obtained after completion of preliminary first two transitions however, for the type (c) transition, since the original flame surface area was doubled, the burning intensity of the Diffusion Flame was equivalent to that of the two Premixed Flame combined. Delichatsios [4] worked on turbulent jet flame and presented an analysis for the transition characteristics from momentum to buoyancy-controlled turbulent jet diffusion flames. A new correlation was derived and compared with experiments for flame heights in turbulent diffusion jet flames ranging from buoyancy to momentum-dominated cases. The results were used to delineate various regimes characteristic of jet diffusion flames such as laminar, turbulent, buoyancy-dominated, momentum-dominated, and lift-off (turbulent jet) flames. Kashiwagi et. al., [5] carried out experimental and theoretical study on time dependent transition phenomenon over a thermally thin sample in two and three-dimensional configurations in microgravity. Exploration suggested that, localized ignition can be initiated at the centre and thus transition to flame could occur either towards upward or downward or both directions simultaneously with external flow. The work provided useful insight into the difference in the transition between the two and three-dimensional configurations. Matalon [6] investigated intrinsic combustion instabilities in both premixed and non-premixed systems, identifying the roles of differential and preferential diffusion, thermal expansion, and heat losses. The study suggested that, for premixed flames, the hydrodynamic instability resulting from thermal expansion plays a central role and is particularly dominant in large-scale flames. It is responsible for the formation of sharp folds and creases in the flame front and for the wrinkling observed over the surface of expanding flames. In contrast, instabilities in diffusion flames, which give rise to cellular and oscillating flames, are mainly driven by diffusive-thermal effects, with thermal expansion playing a secondary role. Puri and Aggarwal [7] presented a detailed review on the understanding of the structure of partially premixed flames with characterization of flames by two sections - one with a flow field where the equivalence ratio is greater than unity and another with equivalence ratio less than unity. A.R Masri [8] carried out intensive study on stratification of turbulent flames to contribute in understanding the transition phenomenon from laminar to turbulent flames. The study concluded that, in laminar partially premixed flames, concentration gradients enhance the interaction between the rich, stoichiometric and lean parts potentially leading to situations where the flame is more resistant to extinction caused by straining and turbulent partial premixing is ubiquitous in practical combustors where the flames are mostly lifted and the compositional inhomogeneity induced at the flame base can vary in time due to flow instabilities as confirmed by various measurements including those of the Flame Index. 
In recently, Shrivastava, et. al., [9] experimentally investigated smoldering to flaming transition by induced parametric variations viz., separation distance, number of external heat source, symmetrical placements and enclosure area. The work verified the existence of coupled transitions and momentous energy transfer. The advancement of combustion systems and rectification of the issues/hazards necessitates through fundamental understanding of the controlling mechanism. Present scientific improvements have relied profoundly on utilization of the analytical, theoretical, numerical and experimental sciences however, the heterogenous nature of the phenomenon involving non-linear interaction between flow, heat, and mass transfer, using such methods has prevented complete understanding of the phenomena. The present work focuses on simplification of flaming transition phenomenon experimentally using images as a potential resource. The experimentations emphasize on distinguishing quintessential flaming transitional from premixed to diffusion flame and flame response as the function of flame length and intensity using image processing. The work is motivated by the need to enhance flaming transition understanding for practical, functional, industrial and research applications. The specific objectives of the work are:

(a) To investigate the effect of surface orientation and entrainment area on transition phenomenon and observe consequential trends in flame structure and characteristics.

(b) To understand the role of key controlling parameters in flaming transition and establish an empirical relation that forestalls transition phenomena.

\section{Experimental Setup and Solution Methodology}

To address the subject, an experimental setup was upraised comprising of (a) Butane cylinder with nozzle (fig. 1(a)), (b) Orientation reference chart, (c) Height reference chart, (d) Camera, (e)Support enclosure Apparatus; (please see figure 1(b)). Selected nozzle contains 4 holes (diameter $4.87 \mathrm{~mm}$ each) for required air entrainment. The experiments were carried out in normal gravity ( $21 \%$ oxygen concentration) environment. The butane flow rate was ensured constant by keeping regulating knob fixed. To observe the flame transition, the entrainment area effect on flames was noted with systematic division of the critical circle area into 5 equivalent parts along the diameter (Fig. 2.). The entrainment area was closed division by division gradually from fully open to fully close for varying orientations. An optical setup was made, and entire experimentation was video graphed. Each study was carried out for 45 seconds and images were extracted from the videos at $60 \mathrm{fps}$. Proper provisions were made to prevent any external influence on the conduct of experiments. The experimental observations were validated with a numerical code was developed using MATLAB. The code was thoroughly validated with the benchmark flame cases and predicted reasonably well. Following which, it was used to analyse extracted images from different experiments to observe trends.

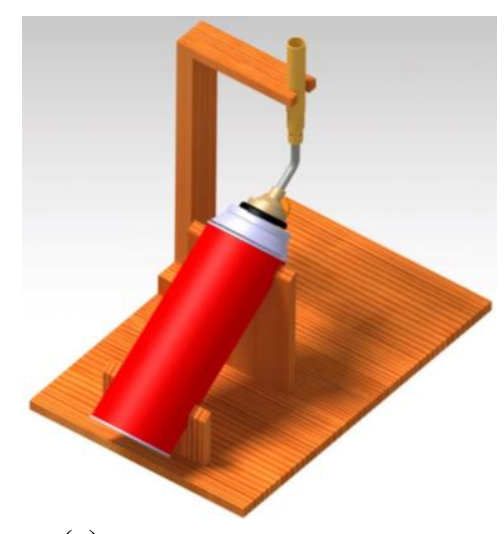

(a)

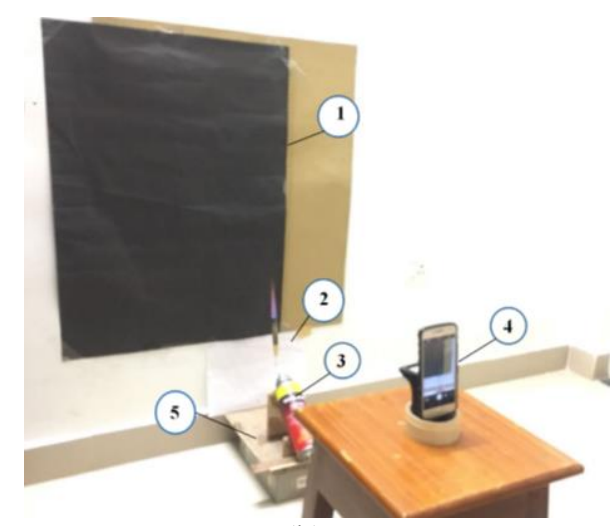

(b)

Fig. 1: (a) Premixed butane gas cylinder with nozzle (b) Complete Experimental setup. 

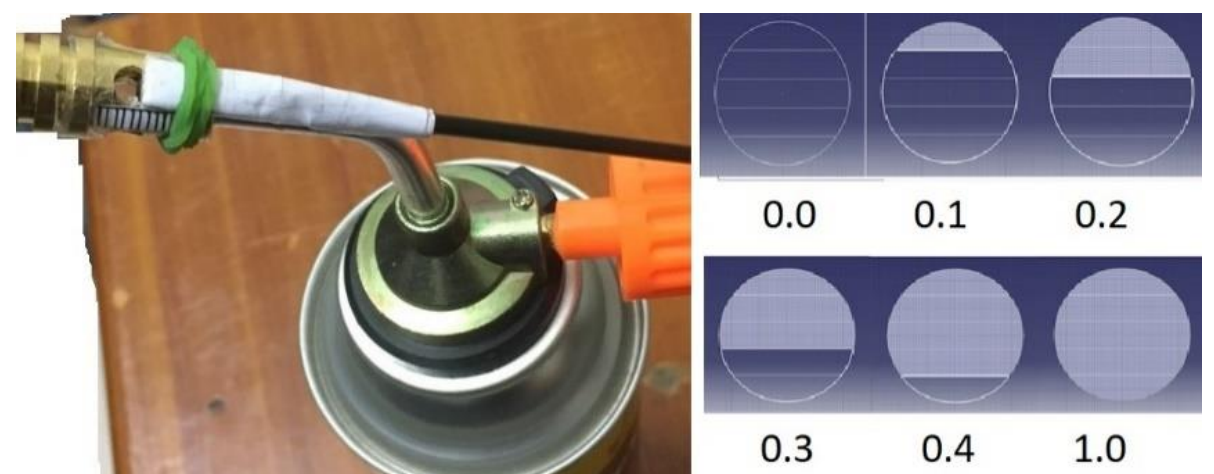

Fig. 2: Manually closing mechanism of entrainment area.

The code specifically performs the following functions: a) tracks the base of flame and helps to verify that mean regression rate is zero. b) Converts the RGB image to grayscale and consequently to binary image through image thresholding based on intensity. c) Calculates average heat release rate for all the images processed. d) Resulting data is then plotted graphically in the form of charts. It is important to note that all the reading presented here represent repeatability.

\section{Results}

Systematic experimentation was carried out in a confined room and at an ambient temperature of $30^{\circ} \mathrm{C}$. First, the occurrence of transition was probed in the selected configuration. Using the manually closing mechanism, the holes on the nozzle were closed stepwise. Additionally, for comprehensive understanding, the tests were conducted at varying orientations. The variation in flame length was investigated thoroughly concurrently with varying enclosed hole configurations. Figure 3 and 4 shows the variation of flame length with variation of enclosure area at varying orientations and figure 5 shows the images for the respective experiments. Completely premixed flame occurs with all nozzle holes open and entirely diffusion flame with all holes closed. The wide-ranging transition was noted to incur with enclosure area associated with 3 holes. Enclosing $1^{\text {st }}$ and $2^{\text {nd }}$ holes yields with gradual changes in the premixing strength whereas, prominent changes in flame shape were observed to evolve with closing of $2^{\text {nd }}$ hole as flame tip changes direction. Fully enclosed configuration results in detailed diffusion flame. For horizontal orientation, in comparison to fully open configuration, minimum changes were observed with closing of $1^{\text {st }}$ and $2^{\text {nd }}$ hole. An abrupt drop in premixing flame strength was noted to occurs with closing of $3^{\text {rd }}$ hole assisted with a ' $M$ ' shaped yellow flames inside blue flame. Flame length was seen to increase by $200 \%$. At 30 degrees inclination, configurations with $1^{\text {st }}$ and $2^{\text {nd }}$ holes enclosed results in $21.429 \%$ increase in flame length however, transition configuration viz., 3 holes enclosure causes $11.765 \%$ decrease. Partial premixing signature were noted in between $2^{\text {nd }}-3^{\text {rd }}$ hole enclosure. While enclosing $4^{\text {th }}$ hole shows flame length increment by $265.38 \% .45$ degrees inclination, represents an interesting case with $10.526 \%$ increment in flame length till $1^{\text {st }}$ enclosed hole followed by from $19.048 \%$ decrement in with $2^{\text {nd }}$ hole closed. Enclosing $3^{\text {rd }}$ hole results in $17.647 \%$ flame length increment trailed by $145 \%$ increase for fully enclosed configuration. 


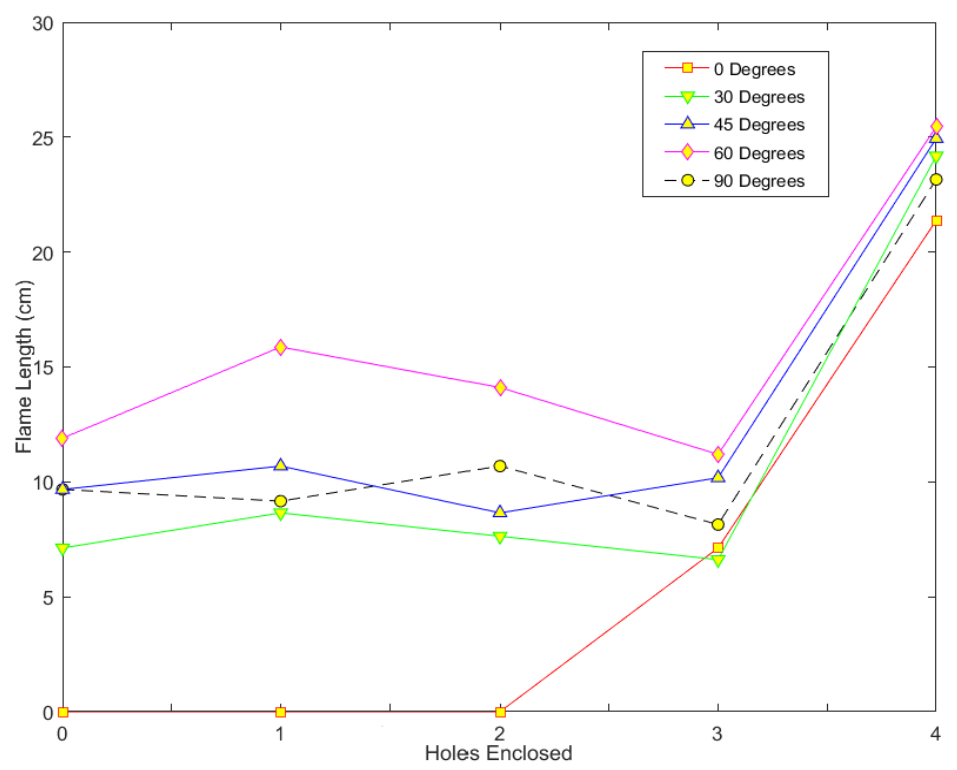

Fig. 3: Variation of flame length with enclosure area for varying orientations.

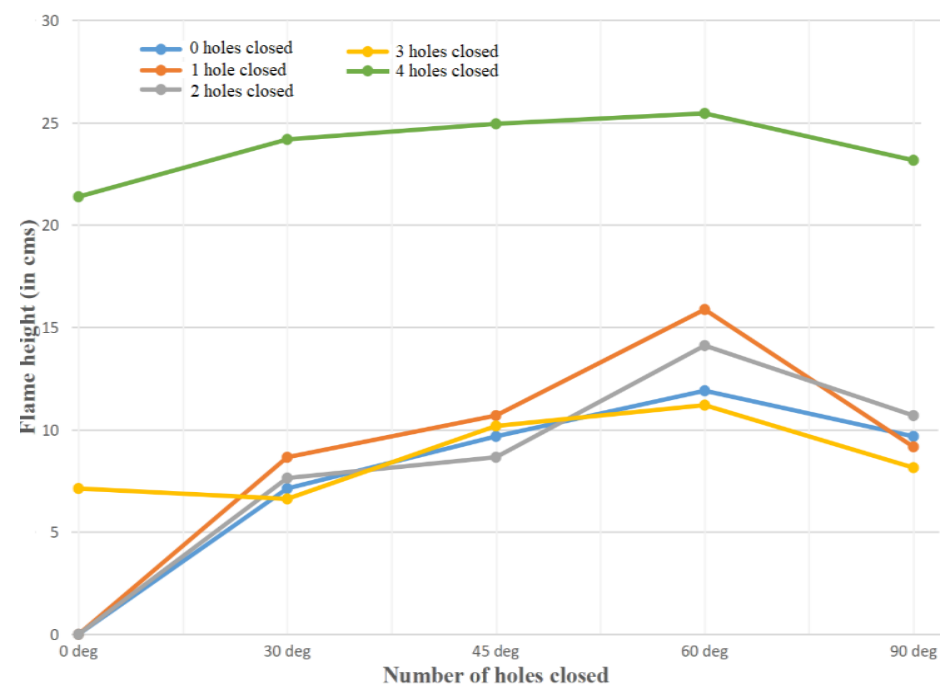

Fig. 4: Variation of flame height with orientation for varying enclosure area. 


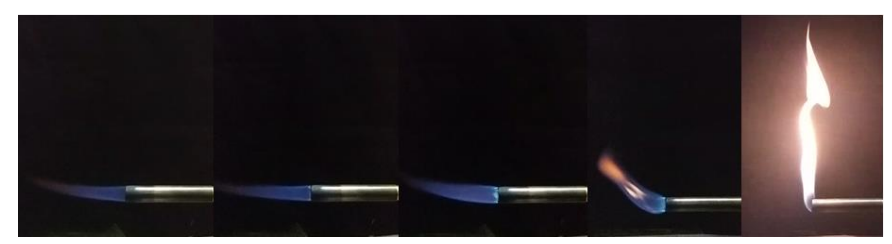

(a)

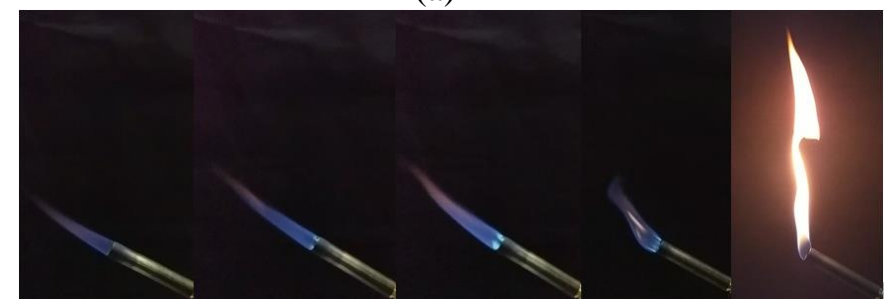

(b)

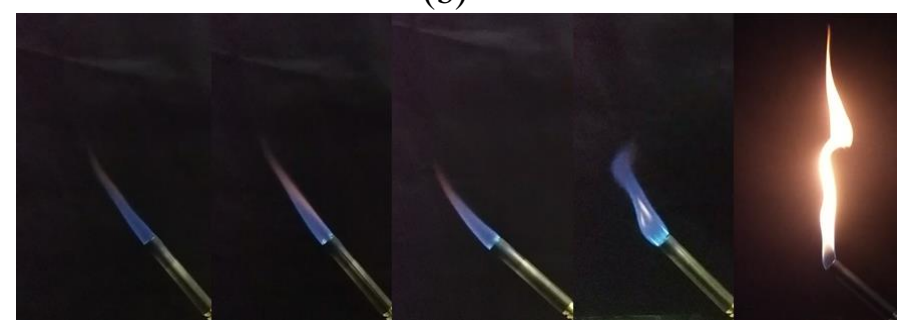

(c)

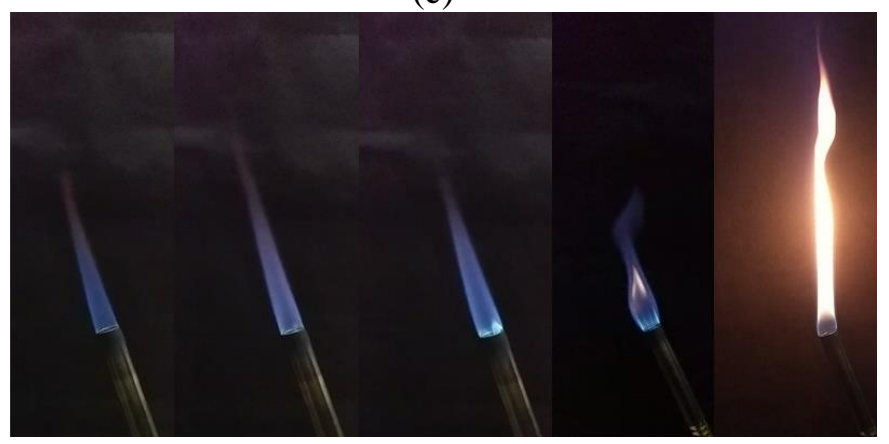

(d)

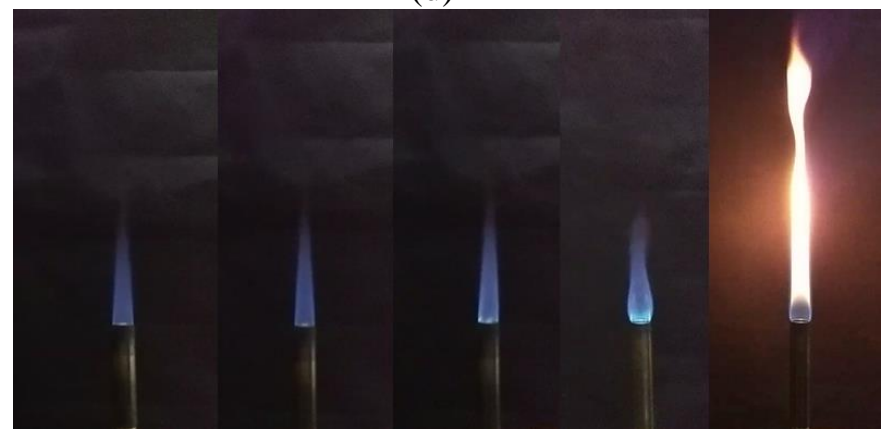

(e)

Fig. 5: Pictorial view of flame shape for varying nozzle orientation (a) 0 degree (b) 30 degrees (c) 45 degrees (d) 60 degrees (e) 90 degrees for varying enclosure area (no. of holes).

60 degrees orientation follows a trend like 45 degrees, $33.344 \%$ increment in flame length occurs till $1^{\text {st }}$ hole enclosure followed by $11.096 \%$ decrement from enclosing $2^{\text {nd }}$ holes. Flame transition configuration marks $20.634 \%$ decrement and finally resulting in $127.27 \%$ increment for diffusion flame configuration. 
Flame height is $9.89 \%$ more than the vertical case and represents the highest flame length among all cases. Vertical orientation results in transitional quantitative trend of flame length with $5.263 \%$ decrement $\left(1^{\text {st }}\right.$ hole) and $16.67 \%$ increment from $2^{\text {nd }}$ hole. Transition configuration highlights $23.8 \%$ decrement and finally resulting in fully diffusion flame with $184.375 \%$ increment. This case is taken as reference for other cases.

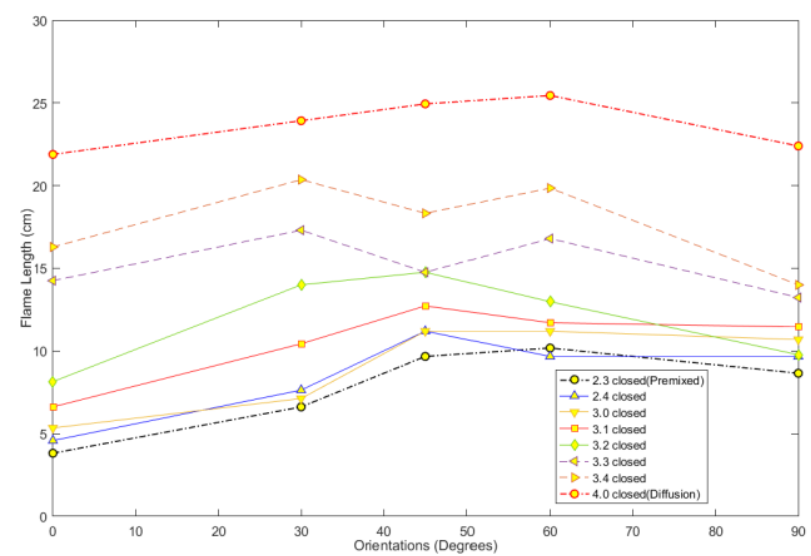

Fig. 6: Variation of flame length with transition $\left(2^{\text {nd }}-3^{\text {rd }}\right)$ hole enclosure area for varying orientations.

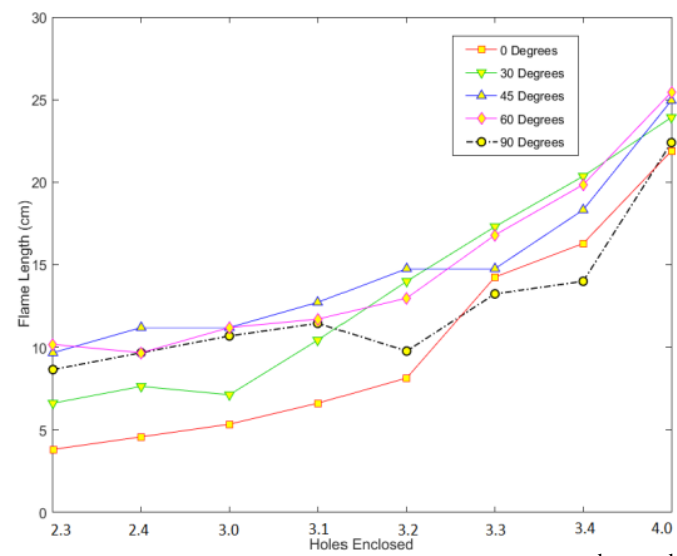

Fig. 7: Variation of flame height with orientation transition $\left(2^{\text {nd }}-3^{\text {rd }}\right)$ hole enclosure area.

As experimentation revealed the flaming transition with $3^{\text {rd }}$ hole enclosure. The $3^{\text {rd }}$ hole was divided into 5 sections (3.04.0) to gain better pictorial depiction along the diameter. Figure 6 and 7 shows the variation of flame length with variation of transition $\left(2^{\text {nd }}-3^{\text {rd }}\right)$ hole enclosure area at varying orientations and figure 8 shows the images for the respective experiments. At 0-degree inclination, steepest slope was observed between enclosed area till sections 2 and 3 of third hole viz., 3.2-3.3. Flame length was noted to abruptly rise by $75 \%$ between $3.2-3.3$ and drops to minimum by $14.285 \%$ between 3.3-3.4. At 30 degrees inclination, linear drop of $6.67 \%$ in flame length was noted between 2.4-3.0 followed by maximum rises by $46.428 \%$ was noted between 3.0-3.1. For 45 degrees, constant values of $15.79 \%$ and $16 \%$ rise were observed between 2.3-2.4 and 3.1-3.2 followed by maximum rise of 36.11\% between 3.4-4.0 enclosed holes. Trend like 45 degrees was observed for 60 degrees orientation. The flame length was spotted to be comparable from 2.4-3.0 and 3.2-3.3 enclosing holes. The highest flame length reached $13.636 \%$ higher than the vertical case. For vertical orientation, 3.1-3.2 enclosed holes results in drop in flame height is by $14.67 \%$ whereas 3.4-4.0 enclosed holes shows $60 \%$ increment.

To understand the behaviour, next we look at the corresponding images. Fig. 9(a) shows at 0 degrees inclination transition phenomenon begins the earliest among all above cases and in between $2^{\text {nd }}$ and $3^{\text {rd }}$ hole enclosure to be exact 3.280/5, which have $64.405 \%$ of total covered entrainment area. 30 degrees inclination transition phenomenon started at 3.350/5, which have covered $68.692 \%$ of total entrainment area (figure 9(b)). Fig. 9(c) shows at 45 degrees inclination transition phenomenon started at 3.385/5, which have covered $70.656 \%$ of total entrainment area about $3.2857 \%$ less than the vertical case. For 60 degrees, transition phenomenon started at 3.4/5, which have covered $71.442 \%$ of total entrainment 
area (figure 9(d)). Fig. 9(e) shows at vertical orientation, transition was first observed exactly at $3^{\text {rd }}$ hole closed. It covered $75 \%$ of total entrainment area representing the case where transition is delayed because of equivalence ratio being close to stoichiometry. The flame length variation was further verified with variation in flame intensity and critical enclosed entrainment area (ratio of yellow area to total flame area) as depicted in figure 10 and 11 respectively and matches reasonably well. It is important to note that as a system, any changes in the stable flame structure and behaviour may be attributed to the variation in fuel, oxidizer or heat intensity and thus resulting in equivalent energy transfer. As noted for above mentioned cases, the reduced oxidiser flow rate resulting in a rich mixture condition ensuing more amount of diffusion flame associated with relatively lower temperature than the premixed flames. The temperature drop indicates heat energy loss compensated by the varying flame length and intensity. Looking at the images, one can note that premixed flames are well behaved under varying conditions as comparable to unstructured diffusion flame pattern.

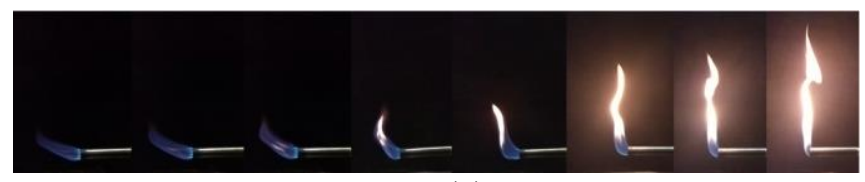

(a)

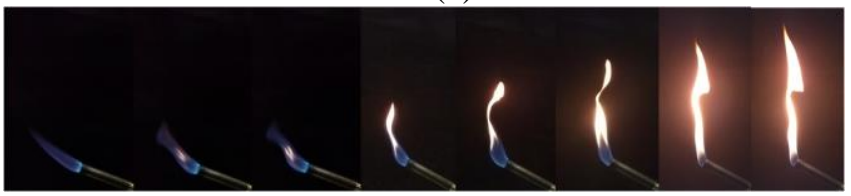

(b)

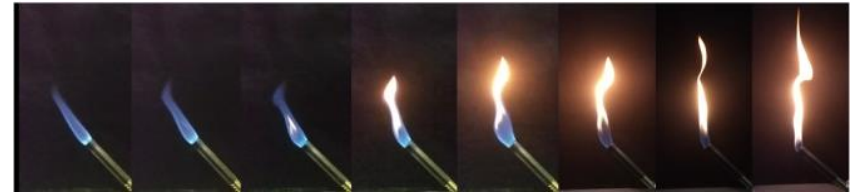

(c)

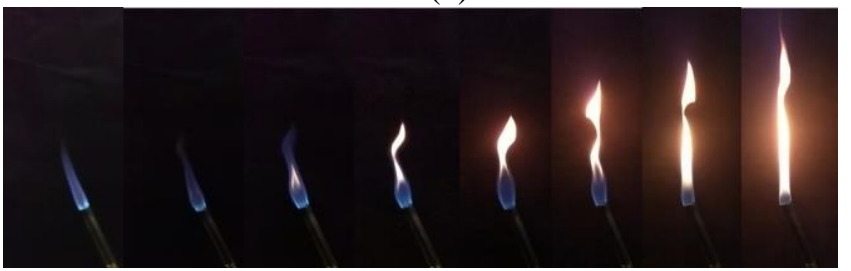

(d)

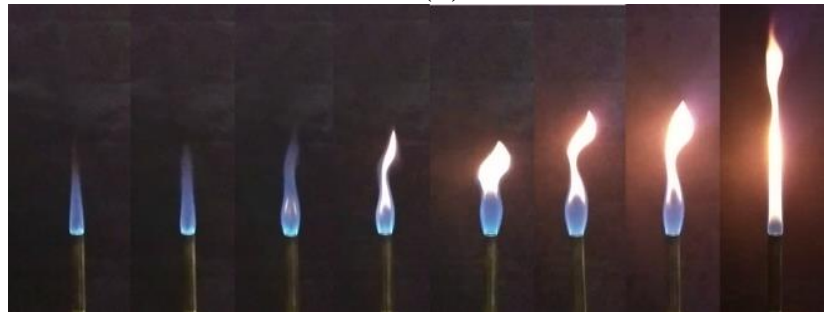

(e)

Fig. 8: Pictorial view of flame shape with varying nozzle orientation for varying $\left(2^{\text {nd }}-3^{\text {rd }}\right)$ hole enclosure area (a) 0 degree (b) 30 degrees (c) 45 degrees (d) 60 degrees (e) 90 degrees.
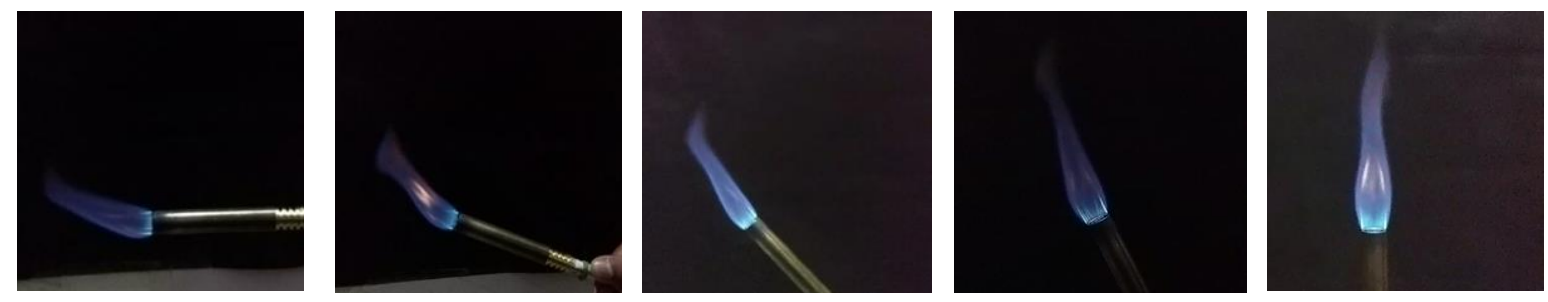
(a)

(b)

(c)

(d)

(e)

Fig. 9: Pictorial view of flame shape with varying nozzle orientation for varying critical transition enclosure area (a) 0 degree (b) 30 degrees (c) 45 degrees (d) 60 degrees (e) 90 degrees.

Careful observation of images shows that, transition forces sudden changes in the stable flame structure as seen by altered flame lengths and intensities. All images show flame transition to develop at same position ( $3^{\text {rd }}$ hole) for all conditions which substantiated that it is an unconditional phenomenon. However, under varying conditions, the transitional flaming behaviour was noted to change signifying that it represents a binomial nonlinearity with varying premixing flame strength. The varying flame structure and emerging manifold flame assemblies within flames can have major implications on the effective utilization of thermal system applications which necessitates significant research emphasis on flaming transition.

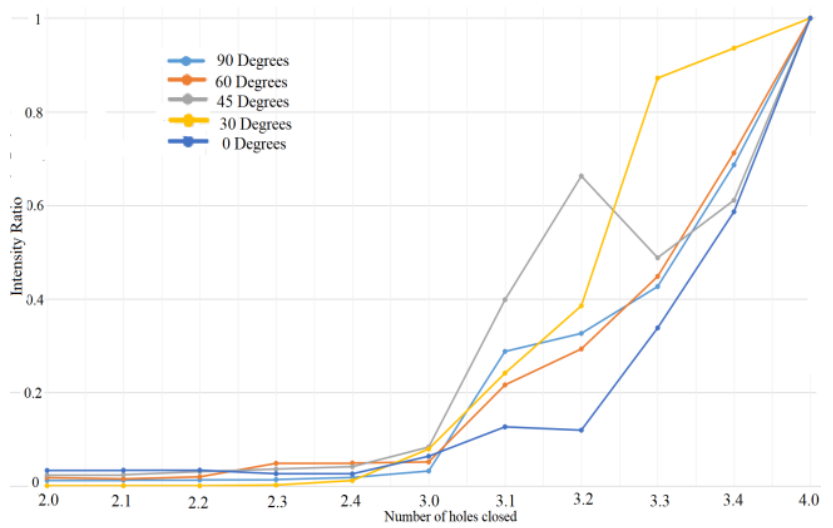

Fig. 10: Variation of flame intensity ratio with enclosed area variation for varying nozzle orientation.

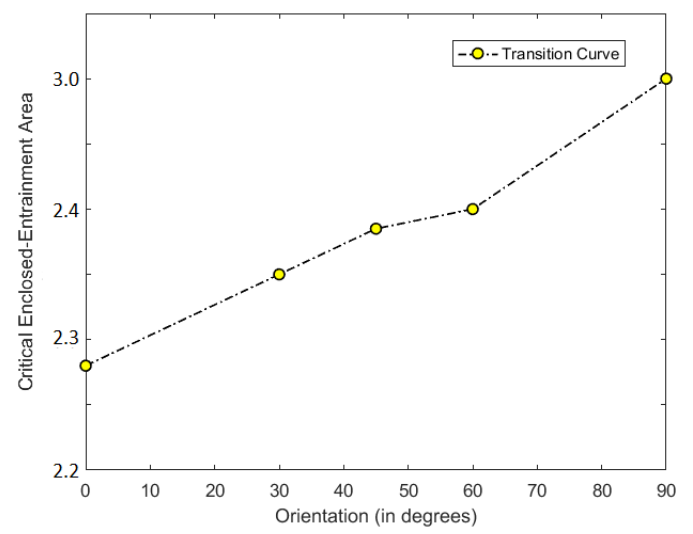

Fig. 11: Induced transition critical enclosed area with nozzle orientation.

\section{Conclusion}

Systematic experimental study was carried out to understand the manually induced flaming transition. The manually induced transition is processed by varying the inlet area for air entrainment. Initialization of the experimental setup details well behaved premixed flames and corrugated diffusion flames. The experimental predictions were verified and validated with appropriate numerical analysis and matches reasonably well. Images acquired from the experiments depict a clear transition representation with related implications. The results obtained clearly quantifies flaming transition as a strong function of enclosure area with linear dependence on orientation. The governing physics associates with strong inter energy conversions leading to rich identification of premixed, transitional and diffusion flames. It is understood from the images that the flaming transition is an unconditional phenomenon, but the implications vary nonlinearly with the conditions system is subjected to accompanied with varying premixing flame strength. The coupled effect may be accountable for reduced combustion efficiency of most of thermal systems and related hazards. 


\section{References}

[1] Von Gerhard Damkohler, "Der Einfluss der Turbulenz Auf Die Flammengeschwindigkeit in Gasgemischen," Z. $f$. Elektroch. Bd., vol. 46. no. 11 (S. 601-652), 1940.

[2] P. H. Thomas, R. Baldwin, A. J. M. Heselden, "Buoyant Diffusion Flames: Some Measurements of Air Entrainment, Heat Transfer, And Flame Merging," Tenth Symposium (International) on Combustion, pp. 983-996, The Combustion Institute, 1965.

[3] T. H. Lin and S. H. Sohrab, "On the Transition of Diffusion to Premixed Flames in Conserved Systems," Combustion and Flame, vol. 68, pp. 73-79, 1987.

[4] M. A. Delichatsios, "Transition from Momentum to Buoyancy-Controlled Turbulent Jet Diffusion Flames and Flame Height Relationships," Combustion and Flame, vol. 92, pp. 349-364, 1993.

[5] T. Kashiwagi, W. Mell, H. R. Baum and S. L. Olson, "Ignition, Transition, Flame Spread in Multidimensional Configurations in Microgravity," $5^{\text {th }}$ International Microgravity combustion workshop, Ohio, 1999.

[6] M. Matalon, "Intrinsic Flame Instabilities in Premixed and Non-Premixed Combustion", Annual Review Fluid Mech., vol. 39, pp. 163-91, 2007.

[7] I. K. Puri and S. K. Aggarwal, "Partially-Premixed Flames: Applications and Issues," $45^{\text {th }}$ AIAA Aerospace Sciences Meeting and Exhibit, Reno, Nevada, 2007.

[8] A. K. Masri, "Partial premixing and stratification in turbulent flames," Proceedings of the Combustion Institute (35), pp. 1115-1136, 2015.

[9] P. Shrivastava, C. Baweja, H. Nalawade, A. Vinoth Kumar, V. Ramanan, and V. Malhotra, "An Experimental Insight into the Smoldering-Flaming Transition Phenomenon," Journal of Combustion, Hindawi Publishing Corporation, vol. 2017, 2017. 